\title{
Why is the Hurricane Season So Sharp?
}

\author{
Wenchang Yang ${ }^{1 *}$, Tsung-Lin Hsieh ${ }^{2}$, Gabriel A. Vecchi ${ }^{1,2}$ \\ ${ }^{1}$ Department of Geosciences, Princeton University, \\ ${ }^{2}$ High Meadows Environmental Institute, Princeton University,
}

*To whom correspondence should be addressed; E-mail: wenchang @ princeton.edu.

This paper is a non-peer reviewed preprint submitted to EarthArXiv. 


\title{
Why is the Hurricane Season So Sharp?
}

\author{
Wenchang Yang ${ }^{1 *}$, Tsung-Lin Hsieh ${ }^{2}$, Gabriel A. Vecchi ${ }^{1,2}$ \\ ${ }^{1}$ Department of Geosciences, Princeton University, \\ ${ }^{2}$ High Meadows Environmental Institute, Princeton University,
}

${ }^{*}$ To whom correspondence should be addressed; E-mail: wenchang@ princeton.edu.

Understanding tropical cyclone (TC) climatology is a problem of profound societal significance and deep scientific interest. The annual cycle is the biggest radiatively-forced signal in TC variability, presenting a key test of our understanding and modeling of TC activity. TCs over the North Atlantic (NA) basin, which are usually called hurricanes, have a sharp peak in the annual cycle, with more than half concentrated in only three months (August to October), yet existing theories of $\mathrm{TC}$ genesis often predict a much smoother cycle. Here we apply a novel framework originally developed to study TC response to climate change in which TC genesis is determined by both the number of pre-TC synoptic disturbances (TC "seeds") and the probability of TC genesis from the seeds. The combination of seed and probability predicts a more consistent hurricane annual cycle, reproducing the compact season, as well as the abrupt increase from July to August in NA across observations and climate models. The seed-probability TC genesis framework also successfully captures TC annual cycles in different basins. The concise representation of the climate sensitivity of TCs from the annual cycle to climate change indicates that the new framework captures the essential elements of the TC climate connection. 
One Sentence Summary: TC frequency annual cycle is due to circulation impacts on both pre-TC vortex seeds and TC genesis probability from seeds.

Tropical cyclones (TCs) are a major natural hazard to life and property (1). Therefore, understanding TC frequency variability and change in response to climate forcing (2-4) is not only of fundamental scientific interest but also crucial in practice. Yet, understanding the connection between climate and TC frequency remains challenging (5-8). In fact, we still do not have a satisfying theory even for its annual cycle. A particularly interesting region is the North Atlantic (NA) basin, where TCs are usually called hurricanes (NA TCs and hurricanes are treated as interchangeable hereafter unless otherwise stated). While the large scale environment in which TCs are embedded evolves smoothly from month to month, hurricane activity usually covers only a few months, with the most active three months from August to October (Aug-Oct). In contrast, hurricane activity is much weaker in the other months, even in the early or middle summer when the thermal condition appears to favor hurricane development.

Fig. 1a shows the annual cycles of NA TC monthly frequency averaged over the recent decades (1980-2018) from both observation and climate model (GFDL/HiRAM) historical simulation. The standout of months Aug-Oct is apparent for both observation and simulation. One simple way of measuring the sharpness of the annual cycle is the ratio of Aug-Oct accumulative value to that from all the other months. This sharpness index is almost three in observation (Fig. 1b), which means approximately three quarters of TCs occur in Aug-Oct. It is slightly lower from the HiRAM simulation but is still more than double, equivalent to two thirds of total TCs being in Aug-Oct. Another pronounced feature related to the sharp annual cycle is the abrupt increase of TC frequency from July to August, by more than 100\% in HiRAM and almost 200\% in observation (Fig. 1c). Besides observation and HiRAM, two other GFDL climate models also show qualitatively similar behavior (see Fig. S1 for AM2.5 and Fig. S2 for 
AM2.5C360). In fact, the sharpness of hurricane season has already been noticed in literature as early as in the 1990s (9), and it has also been found that the annual cycle of more intense hurricanes is sharper than that of weaker tropical cyclones (9).

So what determines this peaked shape of annual cycle? One way to understand the control of TC frequency is to relate it to the sea surface temperature (SST) over the NA basin, or its value relative to the tropical $\left(30^{\circ} \mathrm{S}-30^{\circ} \mathrm{N}\right)$ mean SST (5). However, the annual cycles of both SST and relative SST over NA are smooth and approximately sinusoidal, yielding less sharp peaks over Aug-Oct (see Fig. S3). More sophisticated TC frequency theories usually link TC genesis to various forms of TC genesis indices, which estimate the total impact of multiple crucial variables from the large scale background environment. One particularly interesting index is the genesis probability, or probability of TC genesis from pre-TC tropical disturbances or seeds $(3,10,11)$, which is closely linked to the dynamically derived ventilation index (VI) (10). Ventilation process is hostile to TC development, and a larger VI value tends to yield a lower genesis probability.

Lines in Fig. 1a show the annual cycles of genesis probability monthly climatology calculated from both "observation" (ERA5 reanalysis) and the HiRAM simulation. While the probability does have higher values in TC season in general, the peak of its annual cycle is, however, not as sharp as the TC cycle. The sharpness index has a much lower value: close to one for both observation and simulation (Fig. 1b), which predicts a much lower fraction (around half) of the total TCs occur in Aug-Oct than the actual value. Neither does the genesis probability predict the abrupt increase of TCs from July to August (Fig. 1c). In fact, this is a generic problem also shared by many other forms of TC genesis indices (12-16). While improvement can be made by incorporating more predictors from large scale environment into the indices $(17,18)$, it is often achieved through complex statistical model fitting and therefore the physical process behind the improvement is unclear. 
What causes the discrepancy between the NA TC annual cycle and that predicted by the genesis probability theory? One assumption from the probability theory is the constant supply of TC seeds. In other words, the frequency of tropical disturbances that have the potential to develop into TCs (depending on the genesis probability) does not change with time. Mathematically, the number of TCs is proportional to the number of seeds multiplied by the genesis probability:

$$
N_{T C} \propto N_{\text {seed }} \times p(\Lambda)
$$

The probability theory neglects the variation of $N_{\text {seed }}$ and attributes TC variability solely to probability change. However, recent studies suggest that seeds play a crucial role in the response of TC genesis to climate change $(3,11,19,20)$. These studies demonstrated that the probability change alone is not able to explain the diverging responses of TC frequencies to radiative climate forcings from different climate models and numerical experiments, but the result is promising when taking into account the change of seeds. The question is whether the framework of probability and seed combined together can help us better understand the hurricane annual cycle, which, like climate change, is also primarily driven by radiative forcing. While the signal of climate change often involves a large degree of uncertainty, the annual cycle of hurricanes is clearly defined in observations and well simulated in state-of-the-art high-resolution climate models, which is ideal to test the framework of probability and seed.

Fig. 2 shows the annual cycles of TC, probability, seed and the product of seed and probability from observations as well as the three GFDL high resolution climate models. To focus on the annual cycle, all the quantities are normalized by their annual total value. For observations (Fig. 2a), the NA TC annual cycle predicted by the product of seed and probability is greatly sharpened compared to that predicted by probability alone. As a result, the predicted annual cycle by the new framework is much more consistent with the target TC annual cycle. The improvement of the annual cycle prediction can be attributed to the also relatively high value 
of seed number during the TC season, although the seed annual cycle is much more flat. The predicted TC annual cycles by the new framework are also sharper and more correlated to the actual cycles from the three climate model simulations (Figs, 2b-d). As a result, the sharpness index of $N_{\text {seed }} \times p$ largely increases from probability alone and is more consistent with the that of TC (Fig. S4). Additionally, the abrupt change of TC frequency from July to August is also well captured in the new framework (Fig. S5). Notice that while climate model simulations generally capture the observed TC annual cycle, October normalized TC number is higher than the observed, especially for AM2.5 and AM2.5C360. This appears to be linked to the biased seed number from simulations, with their genesis location mainly over the eastern NA basin off the coast of west Africa in HiRAM (Figs. S6 and S7) but western NA basin in AM2.5 and AM2.5C360 (Figs. S8 and S9). What causes this October seed bias needs further examination.

An alternative way to demonstrate the superiority of the probability and seed framework is to compare the scatter plot of TC versus probability to that of TC versus the product of probability and seed (Fig. 3, all quantities are normalized by the annual total value). Notice that data from both observations and all the three model simulations are now put together in the same scatter plot. While the probability can explain the TC annual cycle to some extent, the new framework of probability and seed has at least three advantages: 1) $N_{\text {seed }} \times p$ can explain a larger fraction of variance from TC (0.97 vs. 0.83); 2) the coefficient of normalized TC regressed on the new predictor is close to one; 3 ) the intercept of the regression is close to zero.

The framework of probability and seeds still holds if we add monthly climatology values from the six other major TC basins besides NA, including the eastern Pacific (EA), western North Pacific (WP), northern Indian Ocean (NI), southern Indian Ocean (SI), Australia (AU) and southern Pacific (SP) basins as shown in Fig. 4. Now we look at the scatter plots from observations and the three model simulations separately but include data from different basins. By comparison, the NA TC has the largest single-month normalized TC frequency (in Septem- 
ber), which makes its annual cycle the sharpest. Overall, the framework of probability and seed works much better than the probability framework in both observations and the three climate models.

In this study, we attempt to address the fundamental issue of TC annual cycle, which provides an observationally constrained test on theories of TC climatology. Current genesis probability theory usually predicts a much smoother annual cycle and is difficult to capture the sharpness of the TC season. By taking into account seed variability, we demonstrate that the new framework reproduces the hurricane annual cycle much more consistently than the probability alone, and in particular, is able to capture the sharp hurricane season. It also provides a unified framework to view TC annual cycles from various basins, sources (both observations and model simulations), and numerical experiments.

Previous studies have shown that the probability and seed framework could help explain diverging responses of TC frequency to radiative climate forcing induced by greenhouse gases $(3,11,21,22)$. Here we test and validate that the new framework also works in a different scenario: climatological variations driven by the annual cycle radiative forcing. To the best of our knowledge, this is the first study to apply the framework to explain TC annual cycle. The two-step thinking of TC genesis (i.e. seed genesis and then TC genesis) might help explain the early finding that more intense hurricanes have much sharper annual cycle than do weaker TCs. Assume that disturbances at the very initial stage have a relatively flat annual cycle and eventually develop into intense hurricanes after multiple steps, of which each step is governed by a probability with an annual cycle shape peaking more or less around the TC season. Then the initial relatively flat annual cycle of disturbances would ultimately become much sharper after times a chain of such probabilities (see Fig. S10, which provides a preliminary support to this hypothesis). This is more likely the case for the stage from a TC seed to an intense hurricane since the probability of each step over this stage might share similar relationship with 
the large scale environment. Probabilities in the early stage from the initial disturbance to a seed appear to be governed by different mechanisms (11).

As the probability and seed framework seems to work across a broad range of different climate forcing scenarios, the immediate question arises: does it also work in the case of unforced or internal variability dominated climate variation? For example, can we use this framework to understand different TC frequencies in El Niño versus La Niña years, as well as contributions from seed and probability in extreme TC years? Another question, which is more fundamental, is what controls the variability of seeds? Can we model the seed frequency variability in a similar way as the TC frequency? While wind shear dominates the NA TC genesis probability annual cycle (Fig. S11), our preliminary analysis suggests that vertical velocity variability plays a dominant role in the seed genesis index proposed by Hsieh et al. 2020 (11) (Fig. S12). This may explain why statistical models of TC genesis can be improved by incorporating vertical velocity (18) or instability (17). More comprehensive analysis and examination are needed to address all these important questions. 


\section{References}

1. K. Emanuel, Annual Review of Earth and Planetary Sciences 31, 75 (2003).

2. F. S. R. Pausata, S. J. Camargo, Proceedings of the National Academy of Sciences 116, 7732 (2019).

3. G. A. Vecchi, et al., Climate Dynamics 53, 5999 (2019).

4. W. Yang, et al., Geophysical Research Letters 46, 7690 (2019).

5. T. R. Knutson, et al., Nature Geoscience 3, 157 (2010).

6. K. J. Walsh, et al., Wiley Interdisciplinary Reviews: Climate Change 7, 65 (2016).

7. T. Knutson, et al., Bulletin of the American Meteorological Society 100, 1987 (2019).

8. T. Knutson, et al., Bulletin of the American Meteorological Society 101, E303 (2020).

9. C. W. Landsea, Monthly Weather Review 121, 1703 (1993).

10. B. Tang, K. Emanuel, Bulletin of the American Meteorological Society 93, 1901 (2012).

11. T.-L. Hsieh, G. A. Vecchi, W. Yang, I. M. Held, S. T. Garner, Climate Dynamics pp. 1-20 (2020).

12. S. Camargo, A. H. Sobel, A. G. Barnston, K. A. Emanuel, Tellus A: Dynamic Meteorology and Oceanography 59, 428 (2007).

13. S. J. Camargo, K. A. Emanuel, A. H. Sobel, Journal of Climate 20, 4819 (2007).

14. M. K. Tippett, S. J. Camargo, A. H. Sobel, Journal of Climate 24, 2335 (2011).

15. C. L. Bruyre, G. J. Holland, E. Towler, Journal of Climate 25, 8611 (2012). 
16. C. E. Menkes, et al., Climate Dynamics 38, 301 (2012).

17. M. DeMaria, J. A. Knaff, B. H. Connell, Weather and Forecasting 16, 219 (2001). Place: Boston MA, USA Publisher: American Meteorological Society.

18. B. Wang, H. Murakami, Environmental Research Letters 15, 114008 (2020).

19. S. Yokoi, Y. N. Takayabu, Journal of the Meteorological Society of Japan 87, 525 (2009).

20. T. Li, et al., Geophysical Research Letters 37, n/a (2010).

21. M. Sugi, et al., SOLA 16, 70 (2020).

22. Y. Yamada, et al., Evaluation of the contribution of tropical cyclone seeds to changes in tropical cyclone frequency due to global warming in high-resolution multi-model ensemble simulations, preprint, In Review (2020).

23. K. R. Knapp, M. C. Kruk, D. H. Levinson, H. J. Diamond, C. J. Neumann, Bulletin of the American Meteorological Society 91, 363 (2010).

24. H. Hersbach, et al., Quarterly Journal of the Royal Meteorological Society (2020).

25. M. Zhao, I. M. Held, S.-J. Lin, G. A. Vecchi, Journal of Climate 22, 6653 (2009).

26. C. S. Bretherton, J. R. McCaa, H. Grenier, Monthly Weather Review 132, 864 (2004).

27. N. A. Rayner, Journal of Geophysical Research 108 (2003).

28. L. M. Harris, S.-J. Lin, C. Tu, Journal of Climate 29, 4293 (2016). 


\section{Acknowledgments}

The simulations presented in this article were performed on computational resources managed and supported by Princeton Research Computing, a consortium of groups including the Princeton Institute for Computational Science and Engineering (PICSciE) and the Office of Information Technology's High Performance Computing Center and Visualization Laboratory at Princeton University. Funding: This work is supported by NOAA/OCO (award NA18OAR4310418), NOAA/MAPP (award NA18OAR4310273), and the Carbon Mitigation Initiative (CMI) at Princeton University. Authors contributions: W.Y., T.L.H. and G.A.V. conceived and designed the study. W.Y. performed the analyses and wrote the initial draft of the manuscript. All authors contributed to interpreting results and refinement of the manuscript. Competing interests: The authors declare that they have no competing interests. Data and materials availability: IBTrACS v04 dataset is available from https://www.ncdc.noaa.gov/ibtracs. ERA5 dataset is available from https://www.ecmwf.int/en/forecasts/datasets/reahalysis-

datasets/era5. All model output data used in this study are available upon request, and will also be made available to the public before the publication of this manuscript. All the codes used in the analyses of this study are available upon request, and will also be made available to the public before the publication of this manuscript.

\section{Supplementary materials}

Materials and Methods

Figs. S1 to S17

References (23-28) 

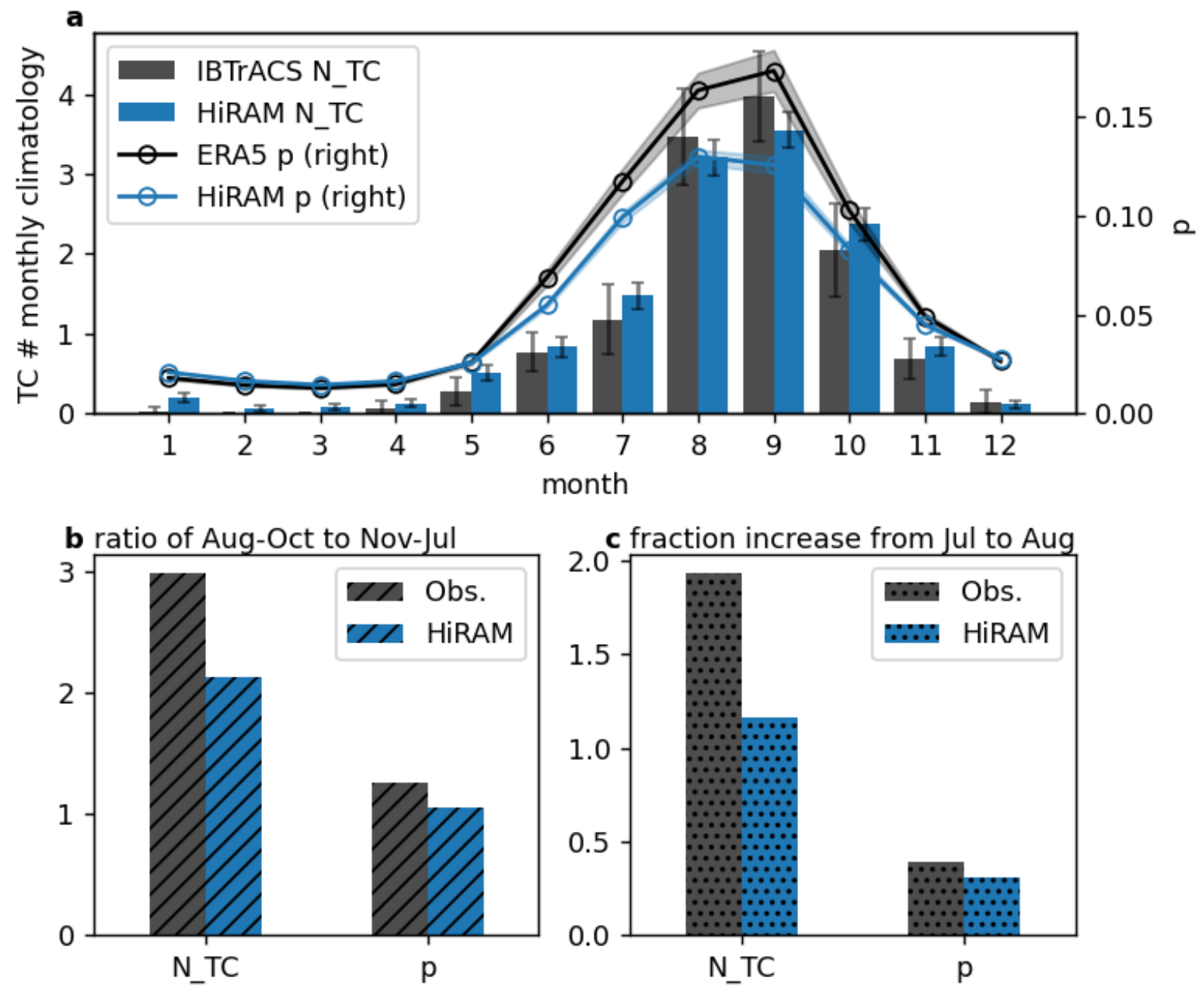

Figure 1: (a): Monthly climatology of NA TC frequency (bars) and genesis probability index (lines) over the years of 1980-2018 from observation and HiRAM simulation. Observed TC frequency is from IBTrACS and genesis probability index is estimated using the ERA5 reanalysis monthly data. Error bars and shading areas indicate 95\% confidence interval of the mean (multi-year mean for the observed, and multi-year-and-ensemble mean for HiRAM simulation). (b): Ratio of accumulated value from Aug-Oct to that from Nov-Jul for TC frequency and genesis probability annual cycles in (a). (c): Fraction increases of TC frequency and genesis probability from July to August in (a). 

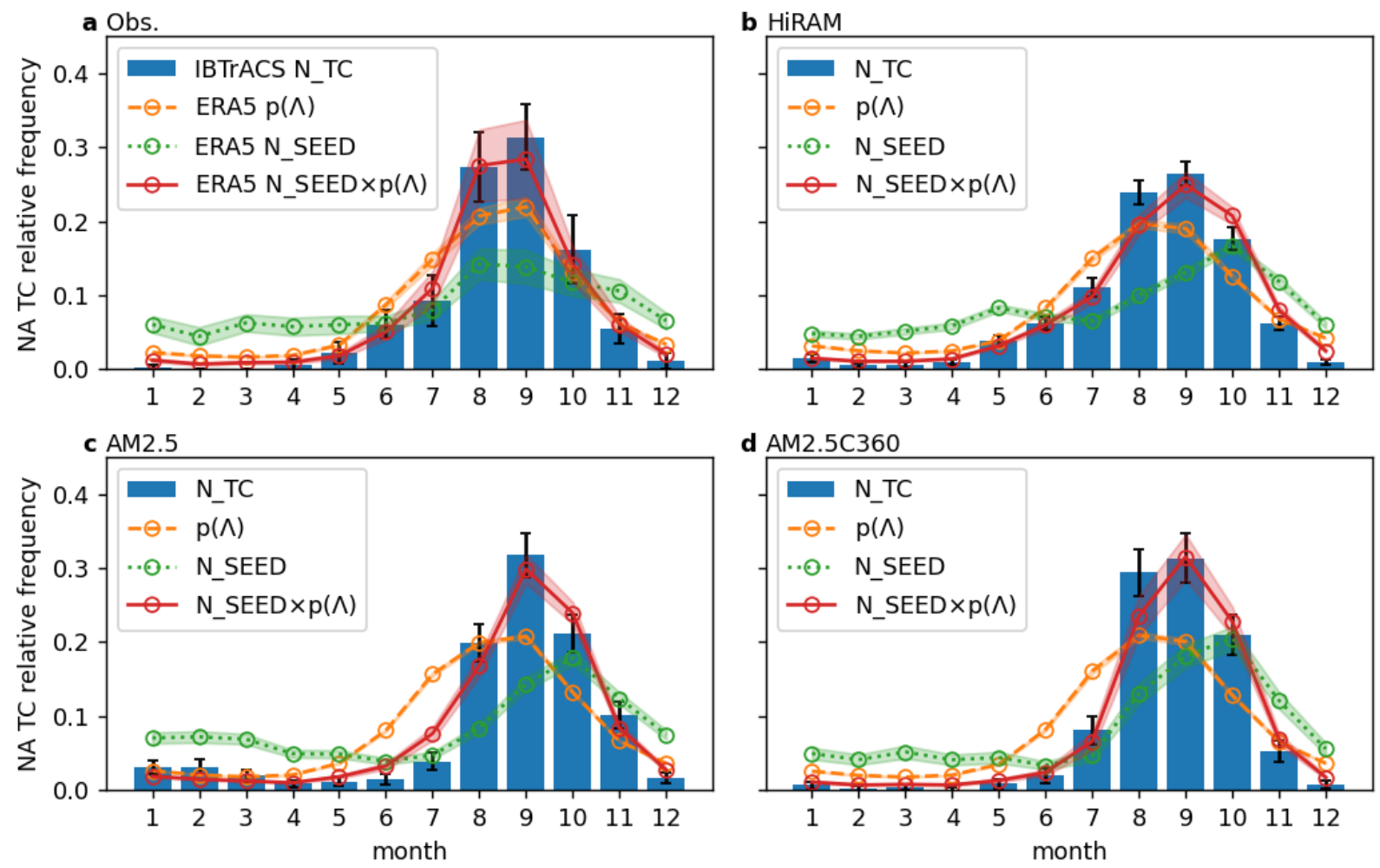

Figure 2: Monthly climatologies of NA TC relative frequency (normalized by the total number of the twelve months, blue bars) and those predicted by TC genesis probability (orange dashed line), vortex seed frequency (green dotted line) and combination of both (red solid line) from observations (a), and model historical simulations from HiRAM (b), AM2.5 (c), and AM2.5C360 (d). Error bars and shading areas show the $95 \%$ confidence interval of the mean. 

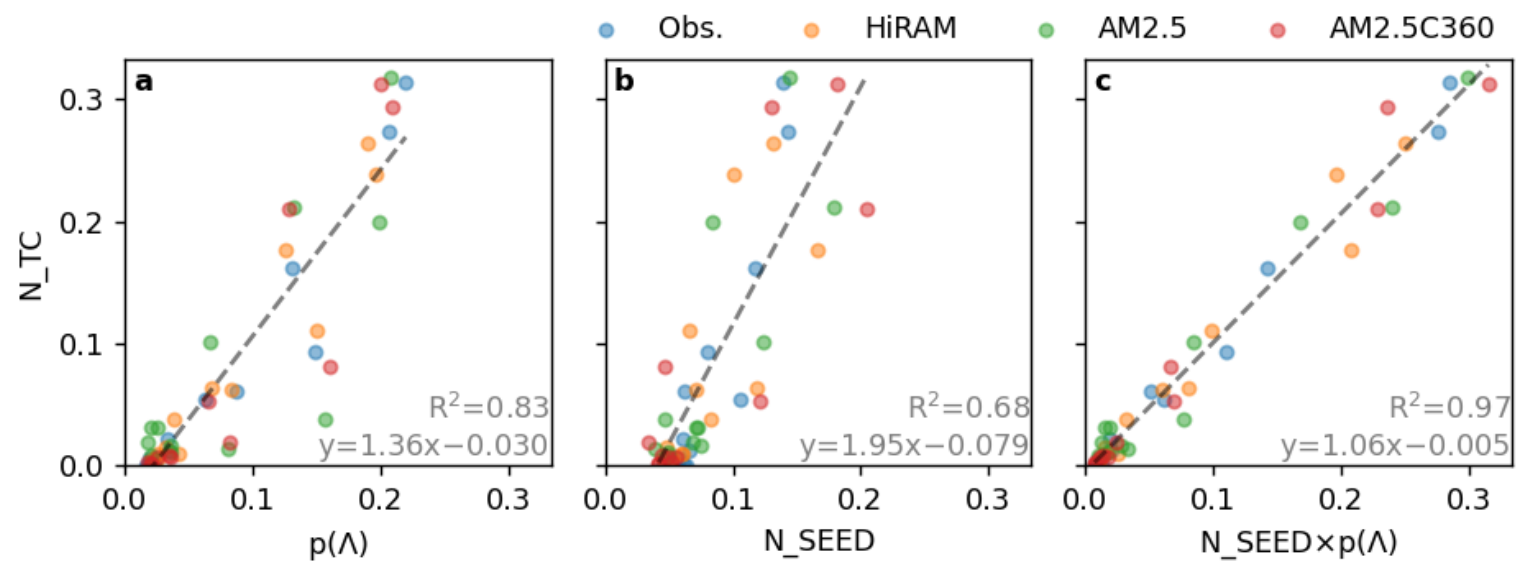

Figure 3: Scatter plots of monthly climatology of NA TC frequency versus TC genesis probability index (left), vortex seed frequency (middle) and the combination of both (right) from observations, model historical simulations from HiRAM, AM2.5, and AMC360. All the quantities are normalized by their annual total. Dashed lines show the linear regression, for which the equation and the variance explained are shown on the bottom right of each panel. 

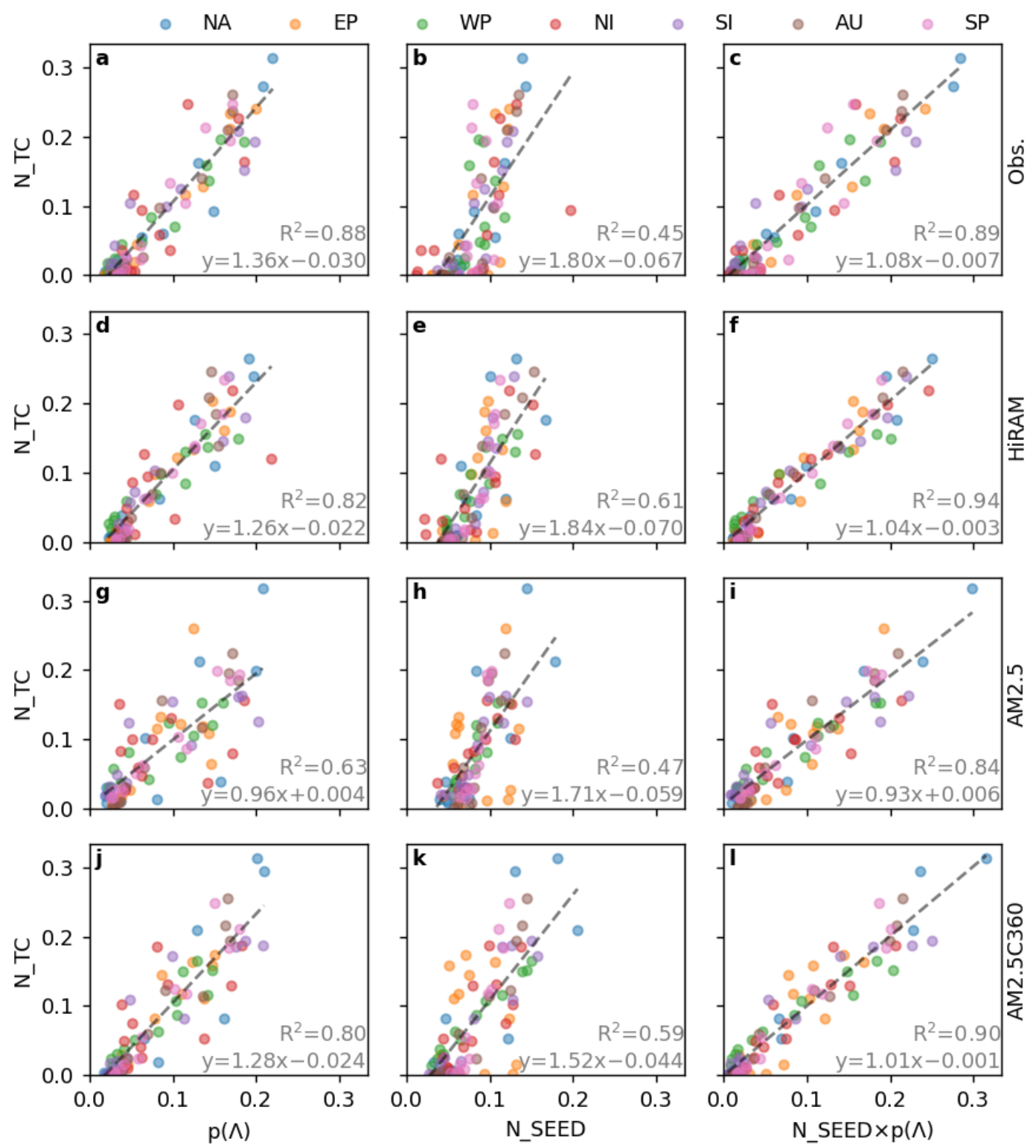

Figure 4: Scatter plots of monthly climatologies of TC frequency versus TC genesis probability index (left column), vortex seed frequency (middle column) and the product of both (right column) over the seven major TC basins of NA, EP, WP, NI, SI, AU and SP from observations (upper row, a-c), and model historical simulations from HiRAM (second row, d-f), AM2.5 (third row, g-i) and AM2.5C360 (bottom row, j-1). Dashed lines show the linear regression, for which the equation and the variance explained are shown on the bottom right of each panel. 


\section{. Supplementary materials}

2 Data.

3 For observed TC tracks, we use version 4 of International Best Track Archive for Climate 4 Stewardship, or IBTrACS v04 (23). The ERA5 reanalysis dataset (24) is used to track seeds of

$5 \mathrm{TC}$, where instantaneous hourly data at UTC hours of $00,06,12$, and 18 ( $4 \times$ daily) are used. We 6 also use ERA5 reanalysis monthly data to calculate large scale climate environment variables 7 and the associated TC genesis probability.

\section{\& Model and numerical experiments.}

9 In this study, we use three AGCMs from GFDL that share the same dynamical core but vary in atmospheric physics or horizontal resolution, including HiRAM (25), AM2.5 and AM2.5C360. HiRAM has a horizontal resolution of about $50 \mathrm{~km}$ and is able to simulate many aspects of the observed TC frequency variability over the past few decades during which reliable observations are available. AM2.5 has the same horizontal resolution of $50 \mathrm{~km}$ as HiRAM but uses the relaxed Arakawa-Schubert convective closure instead of the scheme based on the parameterization of shallow convection from the University of Washington (26) used in HiRAM. $\mathrm{AM} 2.5 \mathrm{C} 360$ is the same as AM2.5 except the horizontal resolution is doubled to about $25 \mathrm{~km}$. Using HiRAM, AM2.5 and AM2.5C360, we performed AMIP-type ensemble simulations (5 ensemble members from HiRAM and AM2.5 and 3 ensemble members from AM2.5C360) in which the atmospheric model is forced by SST from HadISST1 (27) over the period of 19712018. All the ensemble members are forced by the same historical SST and differ only in initial condition. For both observational data and model output from the AMIP historical runs, only years of 1980-2018 are focused on and analyzed unless otherwise stated. 


\section{TC and seed tracking.}

To track TCs, we use the TC tracking algorithm developed by Harris et al. (28), and briefly describe it here as follows. The input data for the tracking algorithm include 6-hourly instantaneous SLP, $850 \mathrm{hPa}$ vorticity, 10-m wind speed and middle-troposphere (300-500 hPa) air temperature. The whole process can be decomposed into two steps. Step 1: storms are first tracked based on SLP, where a maximum $850 \mathrm{hPa}$ cyclonic vorticity magnitude of at least $1.5 \times 10^{-4} \mathrm{~s}^{-1}$ is applied to filter out weak or disorganized systems. Step 2: three lifetime-related conditions are applied on each storm track to get only long-lived TCs. The three minimum lifetimes are: 1) 72 hours of total lifetime; 2) 48 hours of cumulative warm core condition; and 3) 36 consecutive hours of both warm core and maximum 10-m wind speed greater than tropical-storm strength $\left(17.5 \mathrm{~m} \mathrm{~s}^{-1}\right)$. The warm core condition here means the maximum middle troposphere (300-500 $\mathrm{hPa}$ ) temperature is encircled by a $2^{\circ} \mathrm{C}$ (critical temperature difference) contour and is no more than $500 \mathrm{~km}$ (offset radius) from the storm center of SLP.

In our application to the HiRAM TC tracking, we reduce the maximum 10-m wind speed threshold from 17.5 to $15.75 \mathrm{~m} \mathrm{~s}^{-1}$ in the 36 consecutive hours condition but do require the maximum of $10-\mathrm{m}$ maximum wind speed along each storm track is at least $17 \mathrm{~m} \mathrm{~s}^{-1}$. We also modify the warm core condition by increasing the critical temperature difference from 2 to $2.5^{\circ} \mathrm{C}$ and reducing the offset radius from 500 to $110 \mathrm{~km}$. AM2.5 and AM2.5C360 apply the same protocol, except using a critical temperature difference of $1{ }^{\circ} \mathrm{C}$ and the default $2^{\circ} \mathrm{C}$, respectively.

To track vortex seeds, we use the rain cluster tracking algorithm developed by Hsieh et al. (11), which tracks contiguous grid cells whose precipitation rates are larger than the 99.5th percentile of all the tropical $\left(30^{\circ} \mathrm{S}-30^{\circ} \mathrm{N}\right)$ grids at each time step. Clusters are required to be larger than 4 grid points, last longer than one day and initiate within $30^{\circ} \mathrm{S}-30^{\circ} \mathrm{N}$. Seeds are defined as the tracks of the rain cluster whose maximum $850 \mathrm{hPa}$ vorticity along the track 
exceeds $4 \times 10^{-4} \mathrm{~s}^{-1}$ and duration over the ocean surface is at least 12 hours. Our results are generally robust to choice of vorticity or ocean hour thresholds (see Figs. S13-15). We have also tested an alternative seed definition based on SLP that starts from the step 1 of TC tracking described above and further require that the tracks start within $30^{\circ} \mathrm{S}-30^{\circ} \mathrm{N}$ and last at least 12 hours over the ocean surface. The annual cycles predicted using this alternative definition are similar to those using rain cluster (see Fig. S16).

\section{Ventilation index and TC genesis probability.}

Ventilation index is calculated using equation (1) of (10):

$$
\Lambda=\frac{u_{\text {shear }} \chi_{m}}{u_{P I}}
$$

where $u_{\text {shear }}$ is the vertical wind shear between 850 and $200 \mathrm{hPa}, \chi_{m}$ is the entropy deficit and $u_{P I}$ is the potential intensity. Based on the logistic regression model, TC genesis probability is linked to the ventilation index through:

$$
p(\Lambda)=\frac{1}{1+\left(\Lambda / \Lambda_{0}\right)^{n}}
$$

where the parameters of $\Lambda_{0}$ and $n$ are selected to be in agreement with Tang and Emanuel (2012) (10) so that $p(0.014)=0.5$ and $p(0.1)=0.1$. As a result, we get $\Lambda_{0}=0.014$ and $n=\log (9) / \log (0.1 / 0.014) \approx 1.1$

We first calculate monthly TC genesis probability at each lon/lat grid point and then apply area-weighed average between $10^{\circ} \mathrm{N}(\mathrm{S})$ and $30^{\circ} \mathrm{N}(\mathrm{S})$ for the Northern (Southern) Hemisphere basins. We have also tested the sensitivity to the alternative choice in which genesis probability is averaged between $5^{\circ} \mathrm{N}(\mathrm{S})$ and $30^{\circ} \mathrm{N}(\mathrm{S})$ and the result is similar (see Fig. S17). These multiyear (and multi-ensemble for model simulations) basin-mean monthly TC genesis probability time series are thereafter used for the estimation of annual cycles for each basin. 


\section{TC basins.}

Seven global TC basins covered in this study are defined the same as that in Fig. S4 of Yang et al. (4), including the North Atlantic (NA), Eastern North Pacific (EP), Western North Pacific (WP), North Indian (NI), South Indian (SI), Australia (AU), and Southern Pacific (SP) basins.

\section{Normalized annual cycle.}

For TC frequency, genesis probability and seed frequency, the order of processing data is: 1) calculation of monthly time series for each basin; 2) calculation of monthly climatologies; 3) normalization of monthly climatologies by the annual total. For the predictor of $N_{\text {seed }} \times p$, the multiplication takes place between the first and second steps in the above process. 

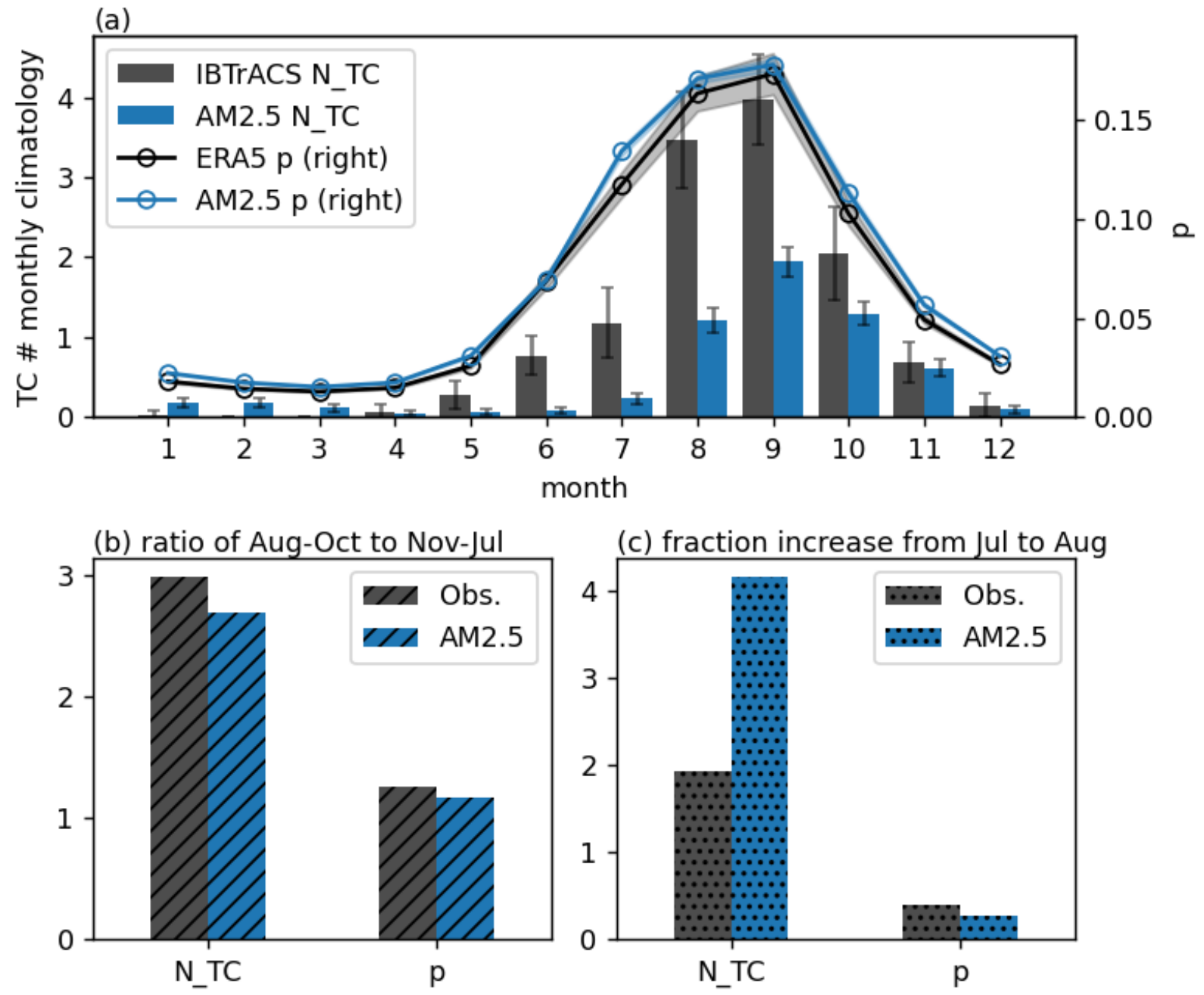

Fig. S1: Same as Fig. 1 except model simulations from AM2.5. 


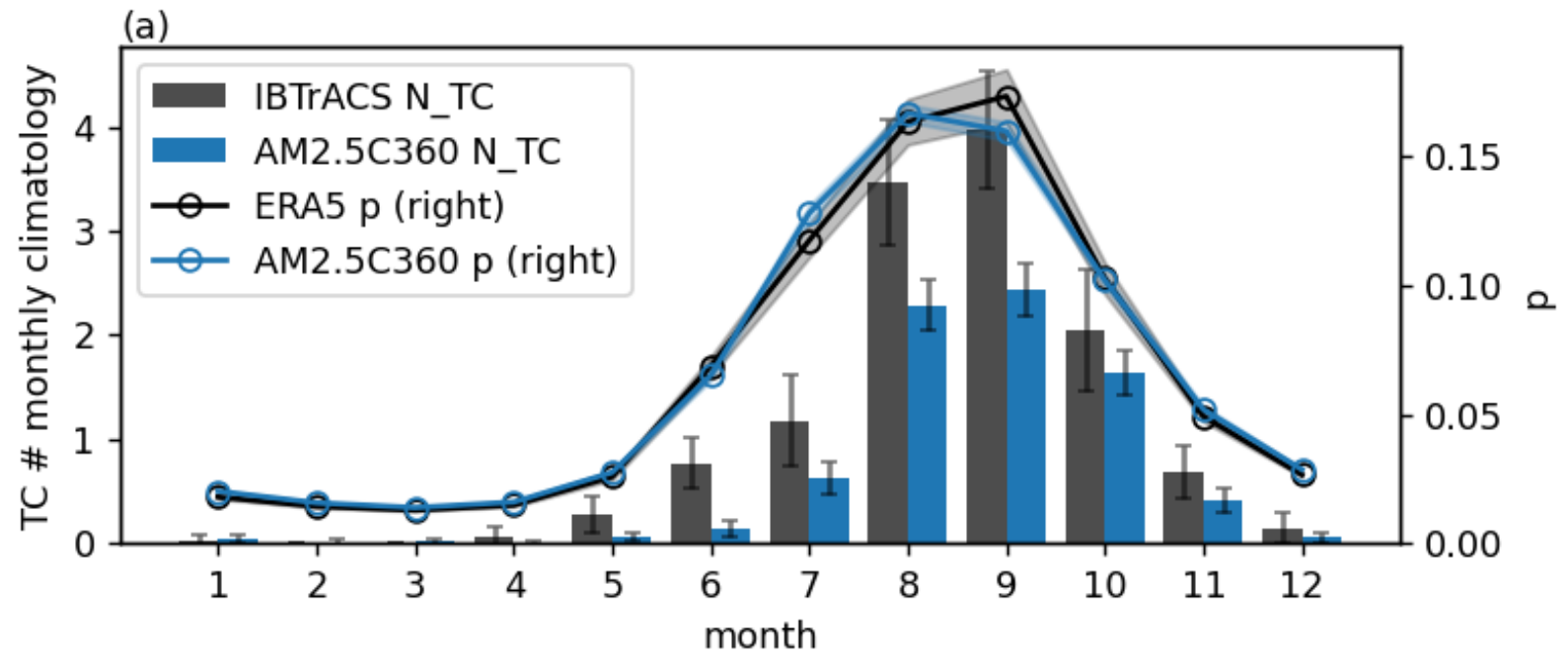

(b) ratio of Aug-Oct to Nov-Jul

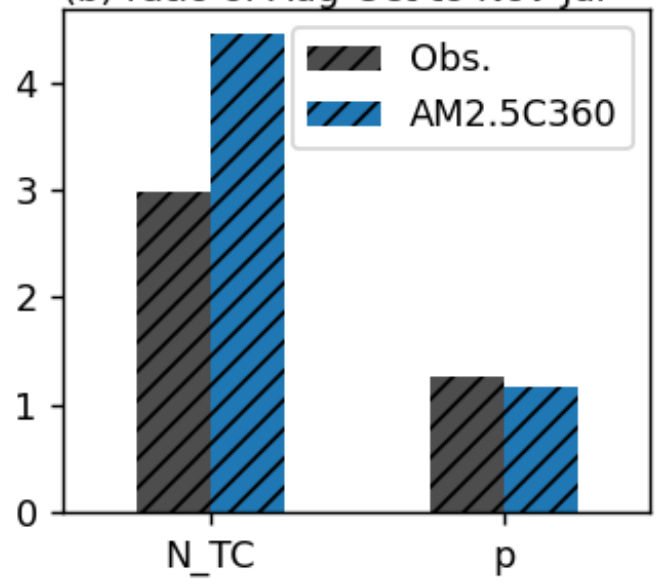

(c) fraction increase from Jul to Aug

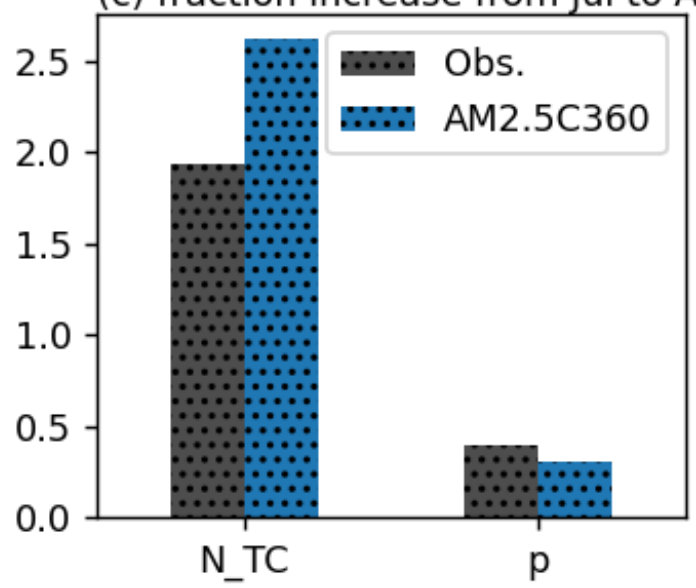

Fig. S2: Same as Fig. 1 except model simulations from AM2.5C360. 


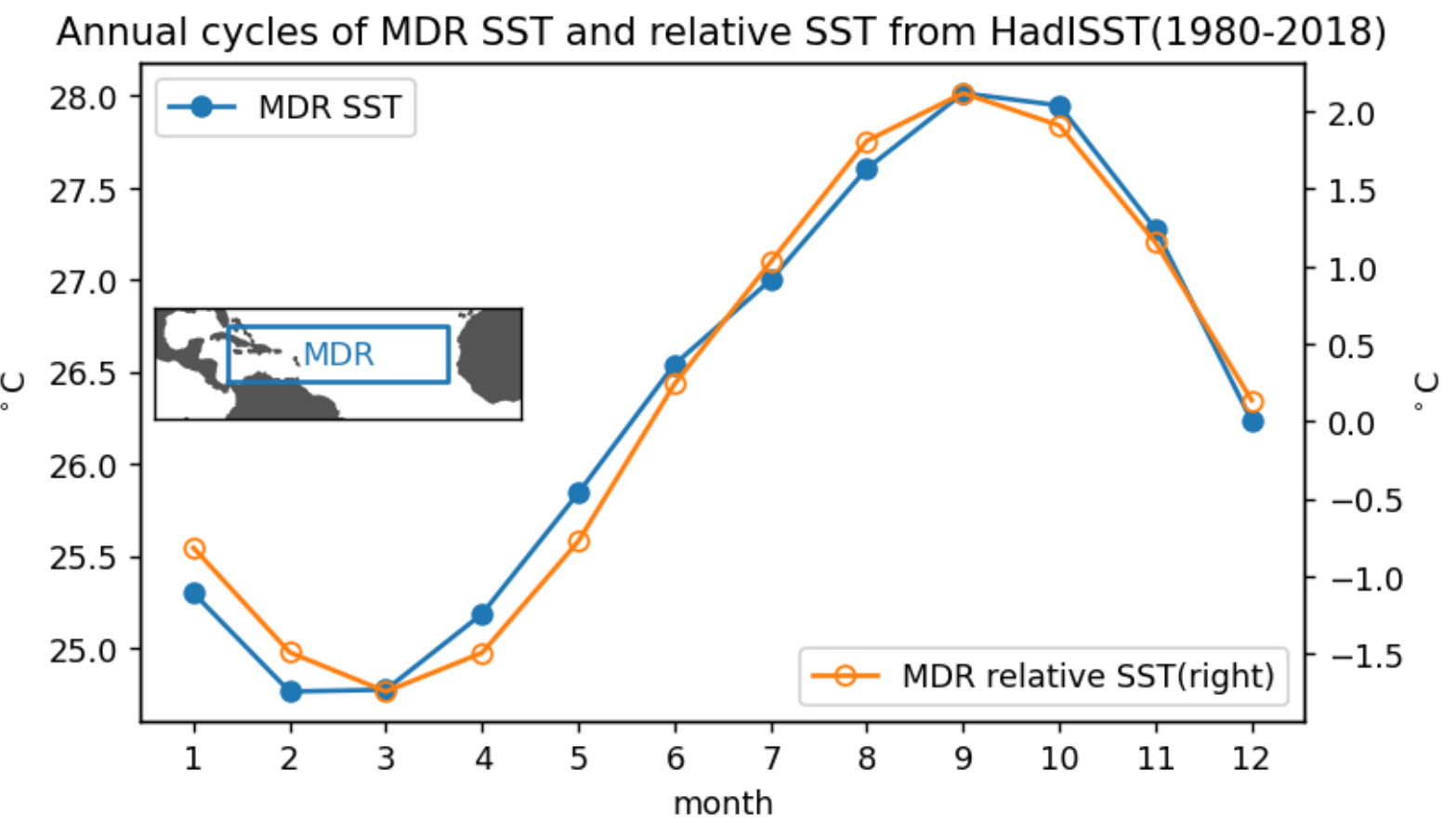

Fig. S3: Annual cycles of SST and relative SST over the Atlantic main development region $\left(\mathrm{MDR}, 10^{\circ}-25^{\circ} \mathrm{N}, 80^{\circ}-20^{\circ} \mathrm{W}\right)$. Relative SST is defined as the anomaly from the tropical $\left(30^{\circ} \mathrm{S}-\right.$ $30^{\circ} \mathrm{N}$ ) mean SST. 


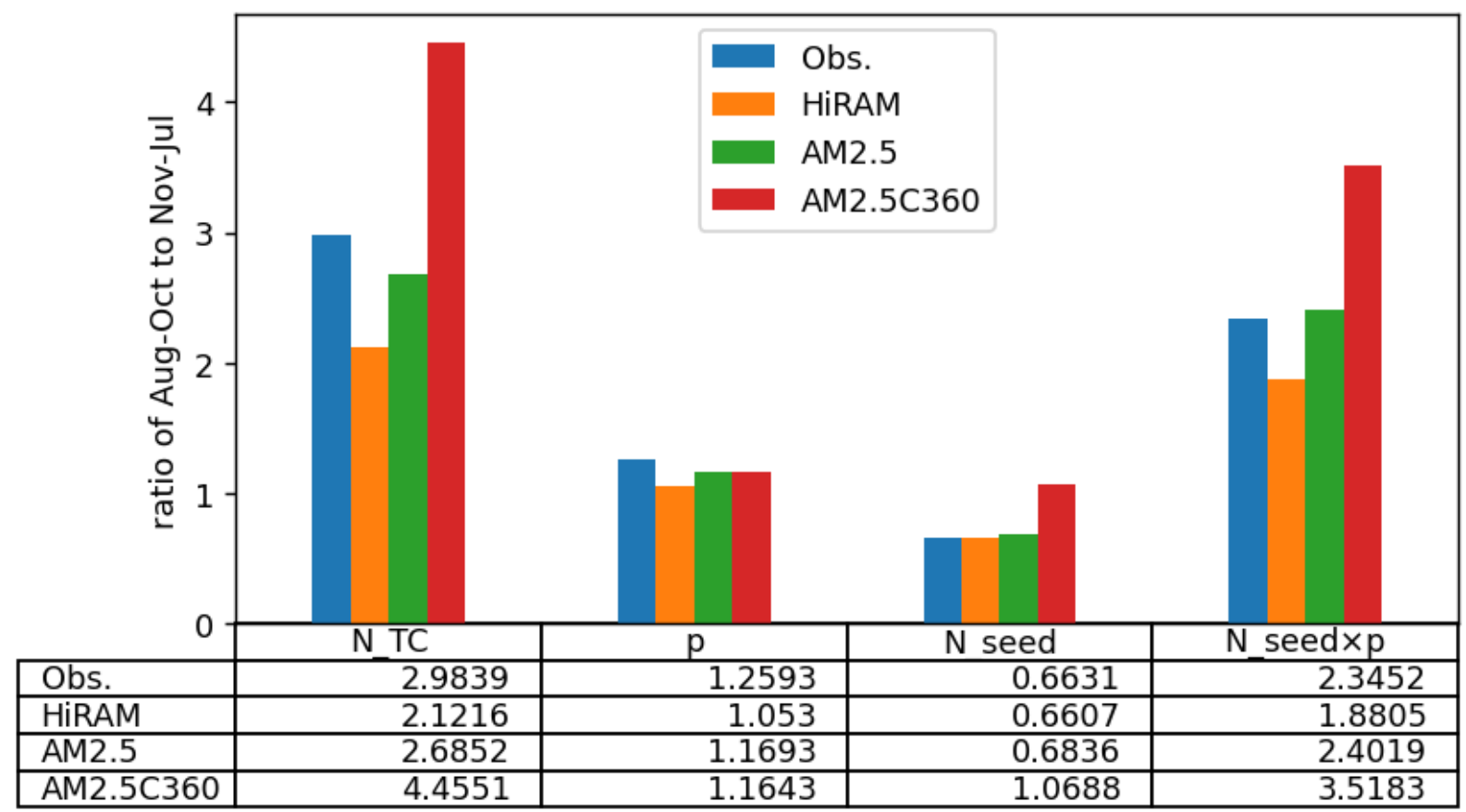

Fig. S4: Ratio of accumulated value from Aug-Oct to that from Nov-Jul for TC number, genesis probability, seed number, and seed number multiplied by probability from both observations and the three climate models. 


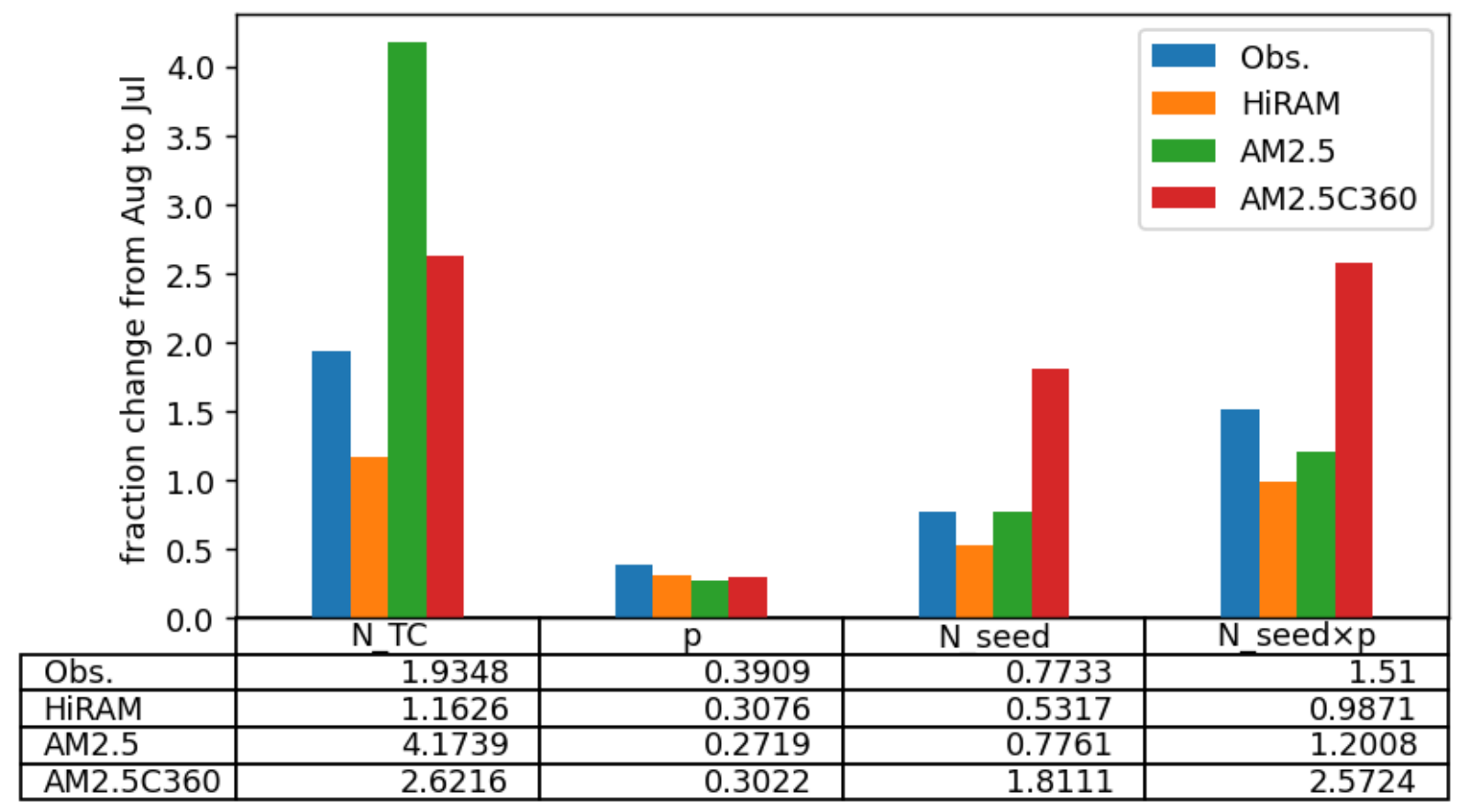

Fig. S5: Fraction changes from July to August for TC number, genesis probability, seed number, and seed number multiplied by probability from both observations and the three climate models. 

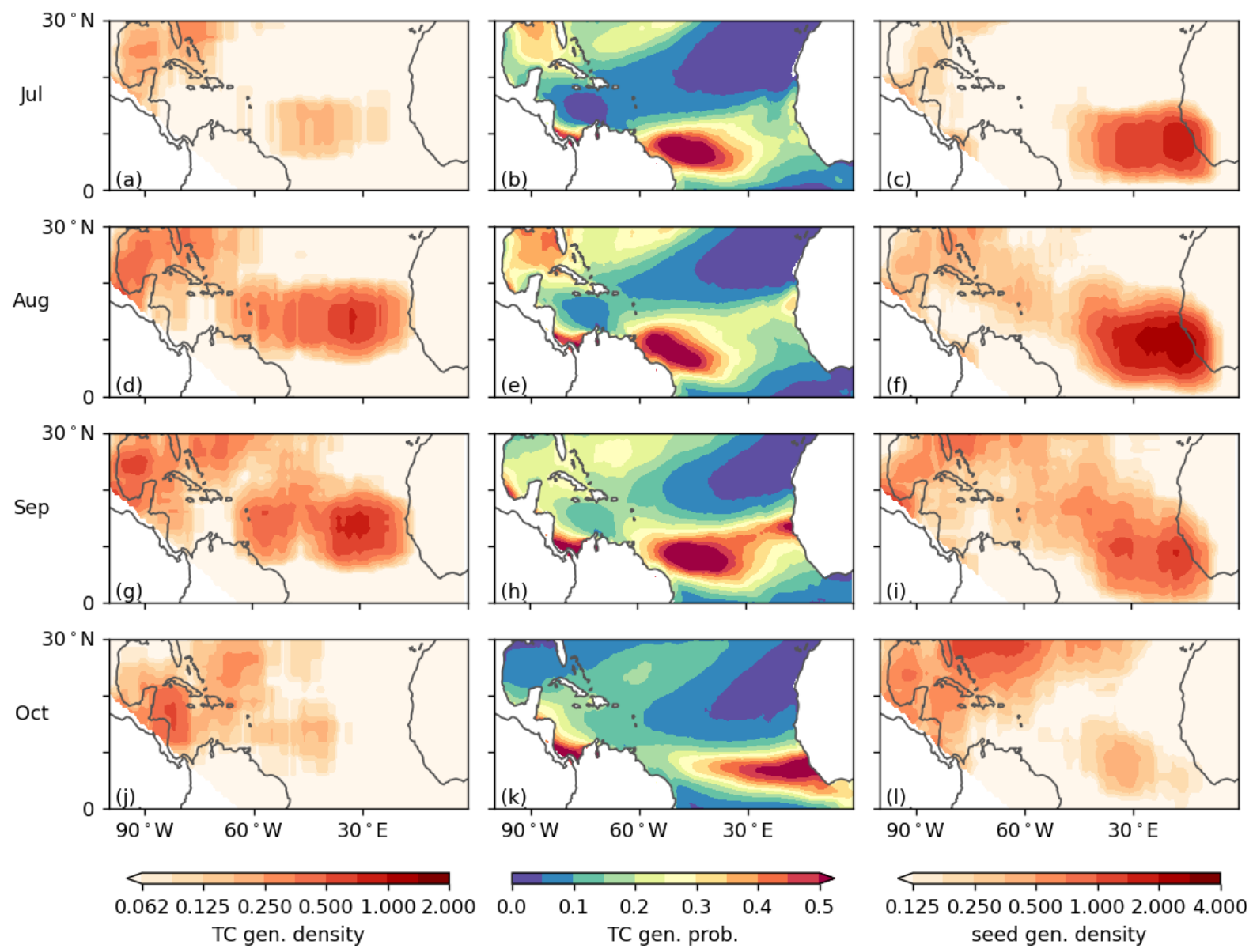

Fig. S6: Monthly climatology maps of TC genesis density (left column), genesis probability index (middle column) and vortex seed genesis density (right column) in July (top row), August (second row), September (third row) and October (bottom row) from observations. TC genesis density is estimated from IBTrACS and the units are: \# per month per $10^{\circ} \times 10^{\circ}$ box. TC genesis probability index and the vortex seed genesis density are estimated based on the ERA5 reanalysis dataset. The units of the vortex seed genesis are also \# per month per $10^{\circ} \times 10^{\circ}$ box. 

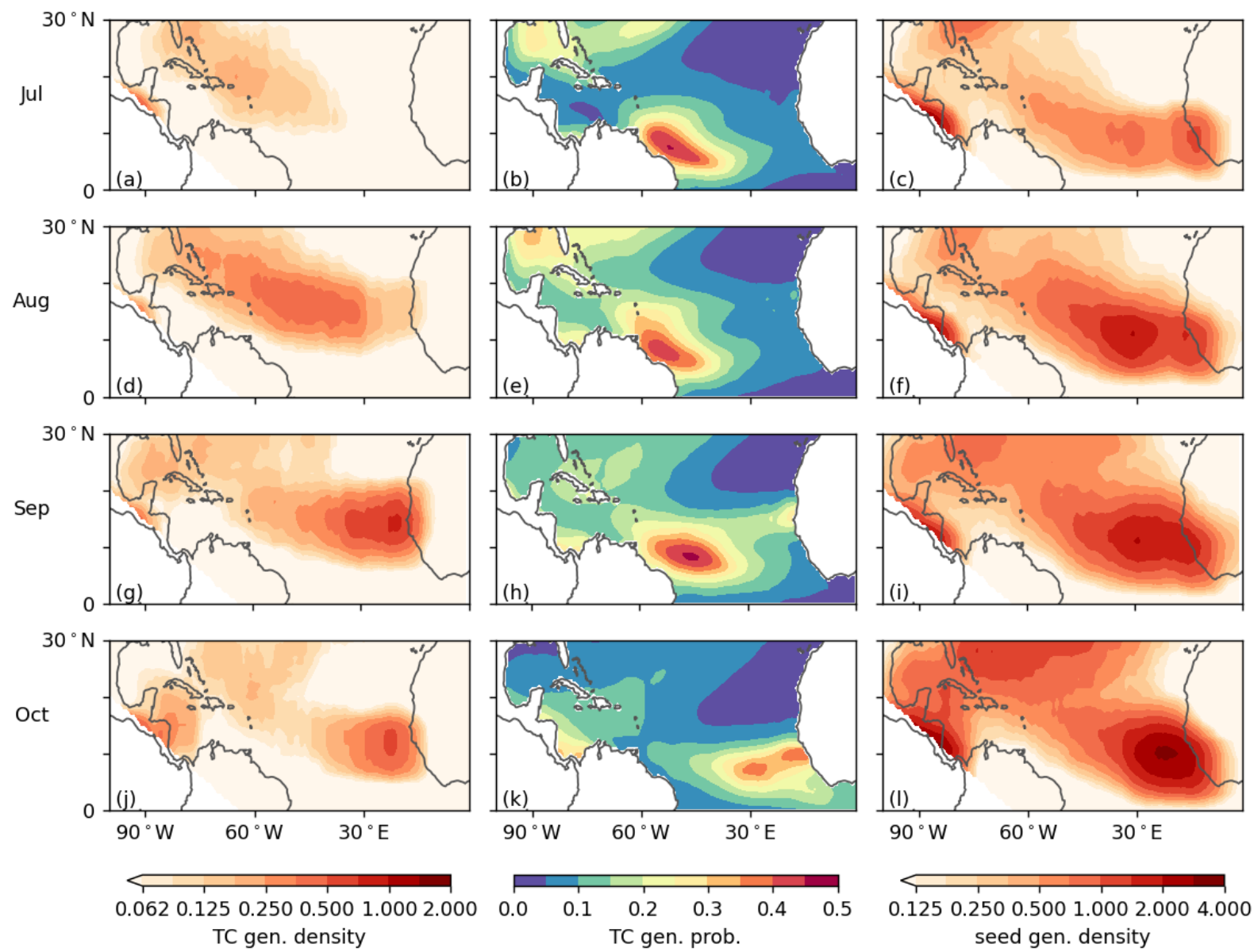

Fig. S7: Same as Fig. S6 except for HiRAM simulations. 

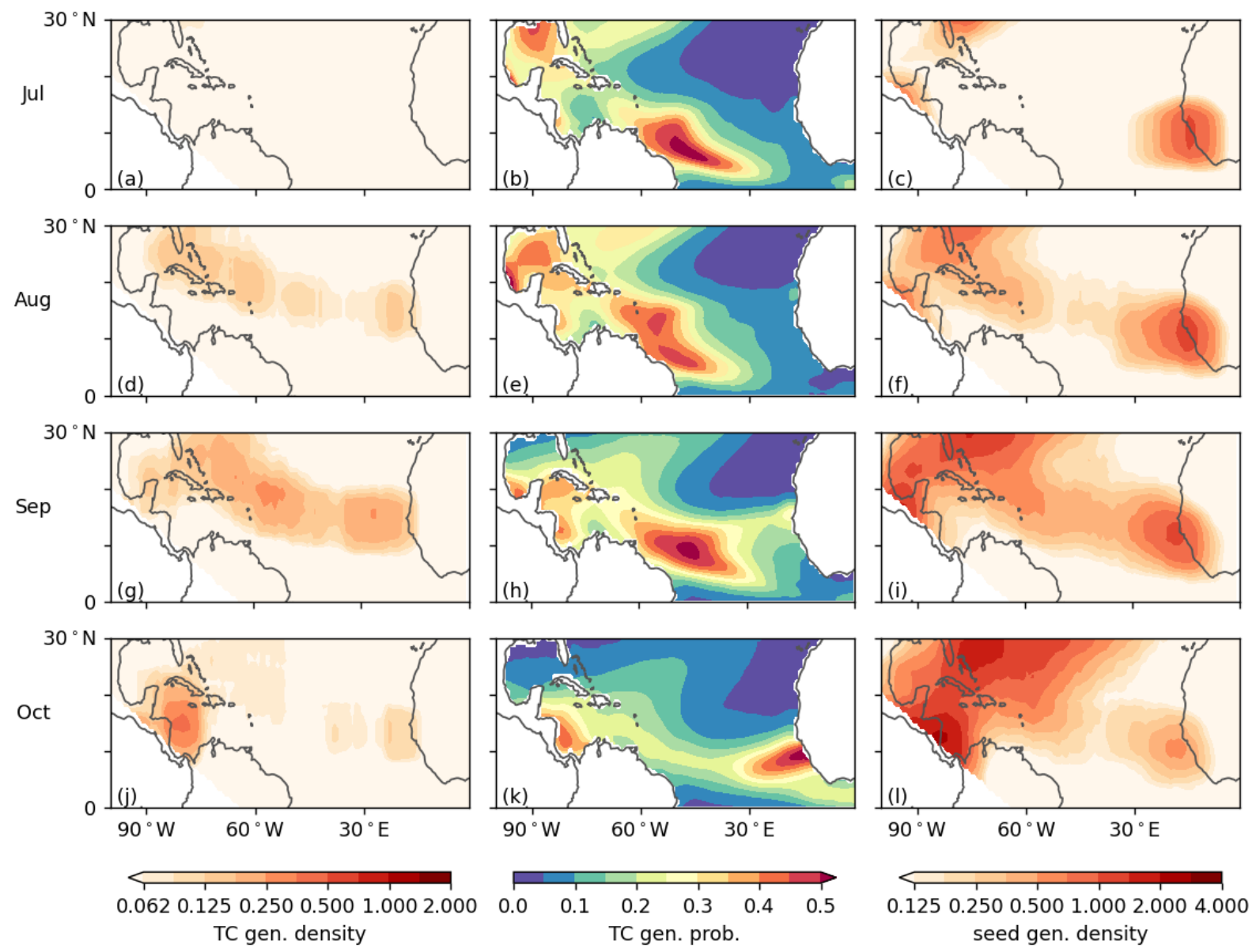

Fig. S8: Same as Fig. S6 except for AM2.5 simulations. 

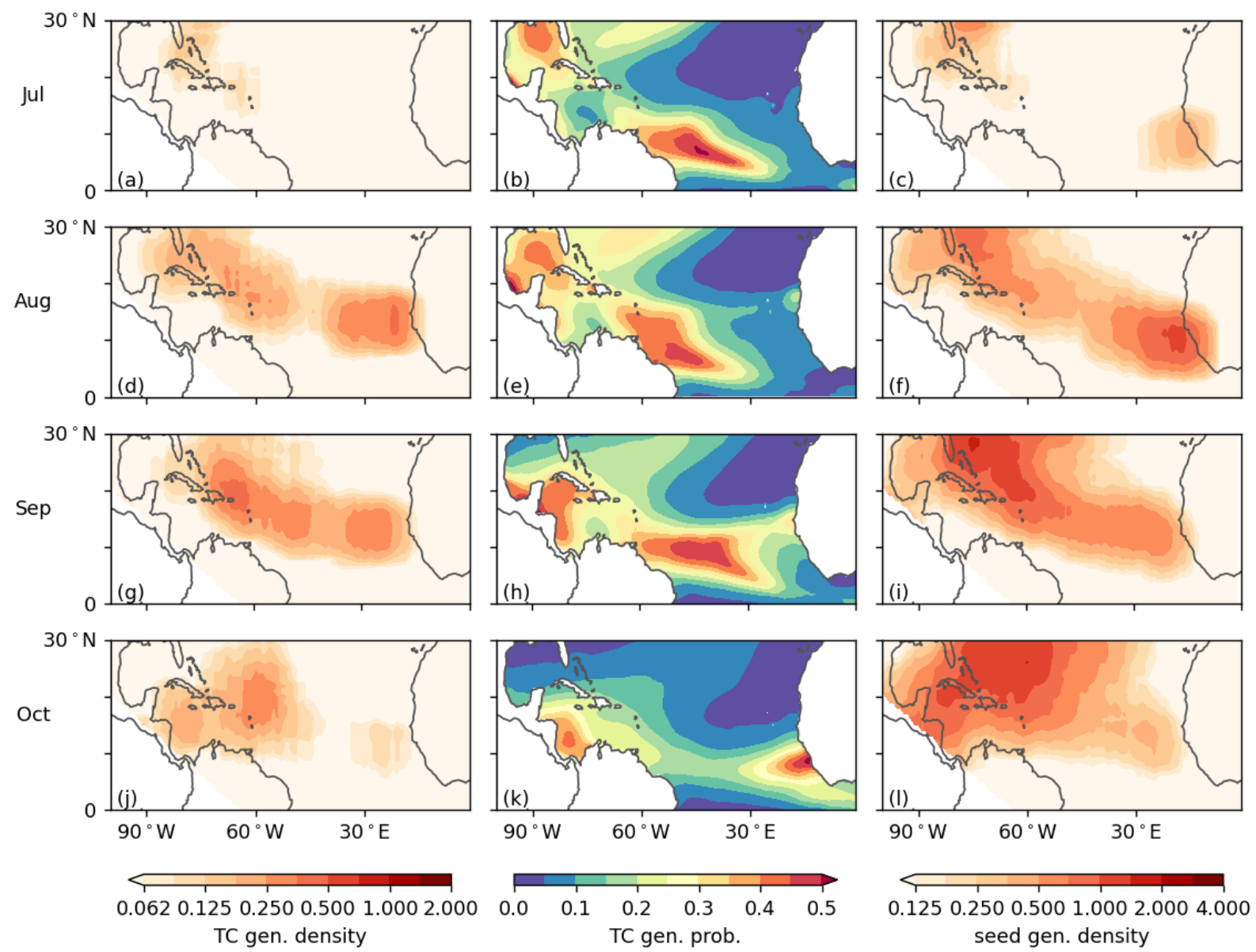

Fig. S9: Same as Fig. S6 except for AM2.5C360 simulations. 


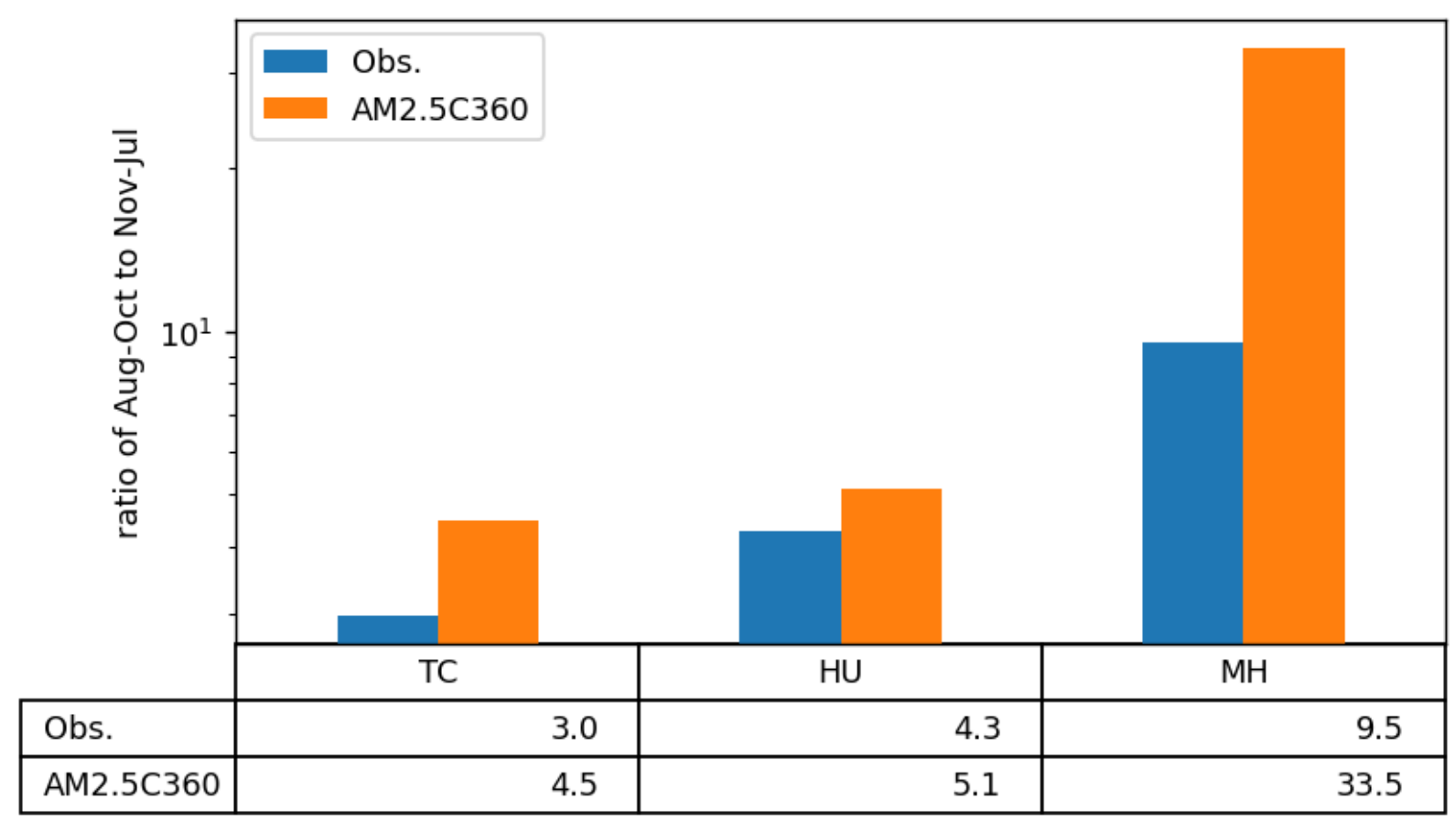

Fig. S10: Ratio of accumulated value from Aug-Oct to that from Nov-Jul for all TCs, Cat1-5 TCs (HU) and Cat3-5 TCs (MH) numbers in observation and the AM2.5C360 climate model. 

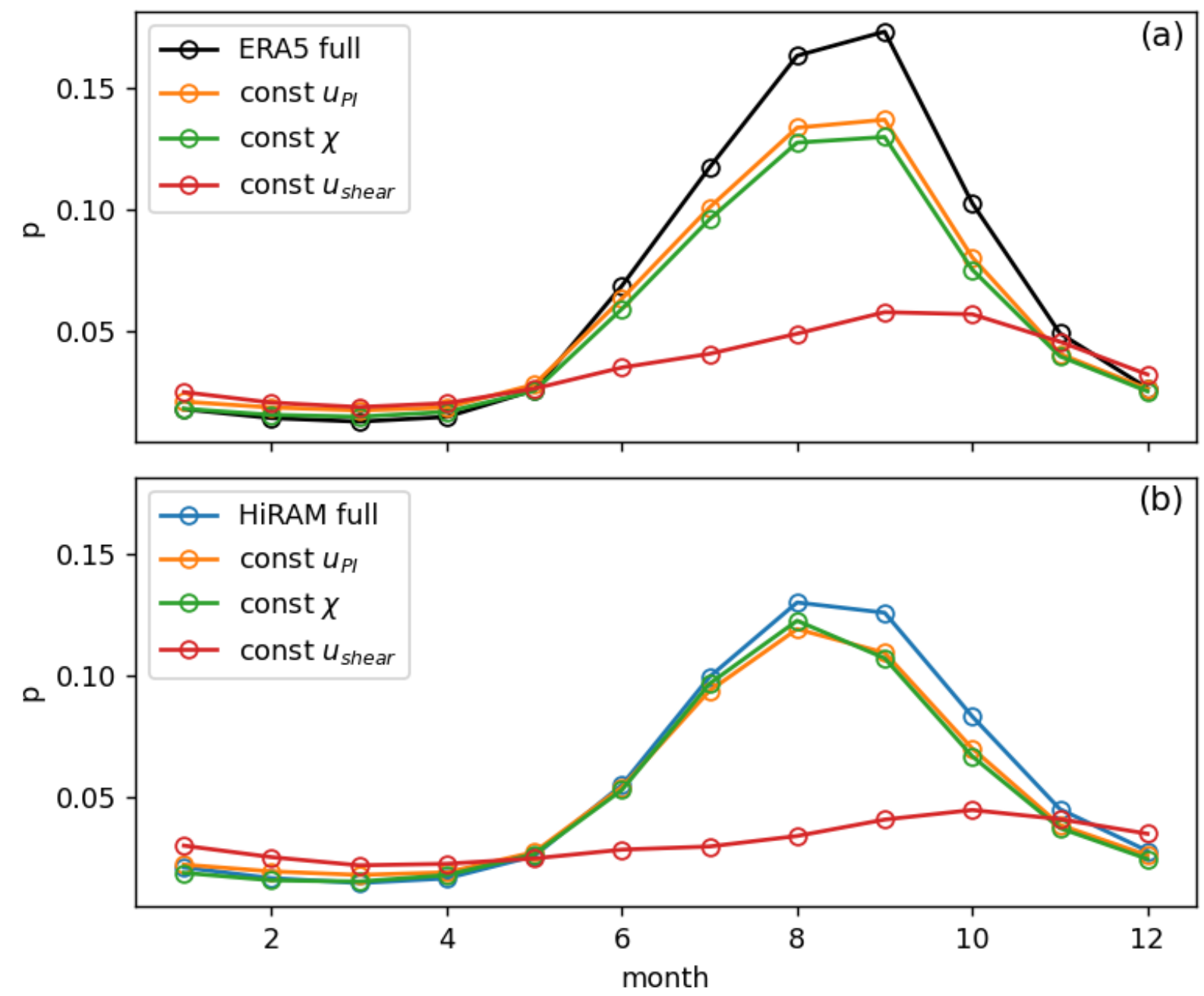

Fig. S11: (a) ERA5 NA TC genesis probabilities calculated using annual mean climatology of potential intensity (orange), entropy deficit (green) and wind shear (red). The actual probability annual cycle from (a) is also shown for comparison (black). (b) Same as (a) but for results from HiRAM. 


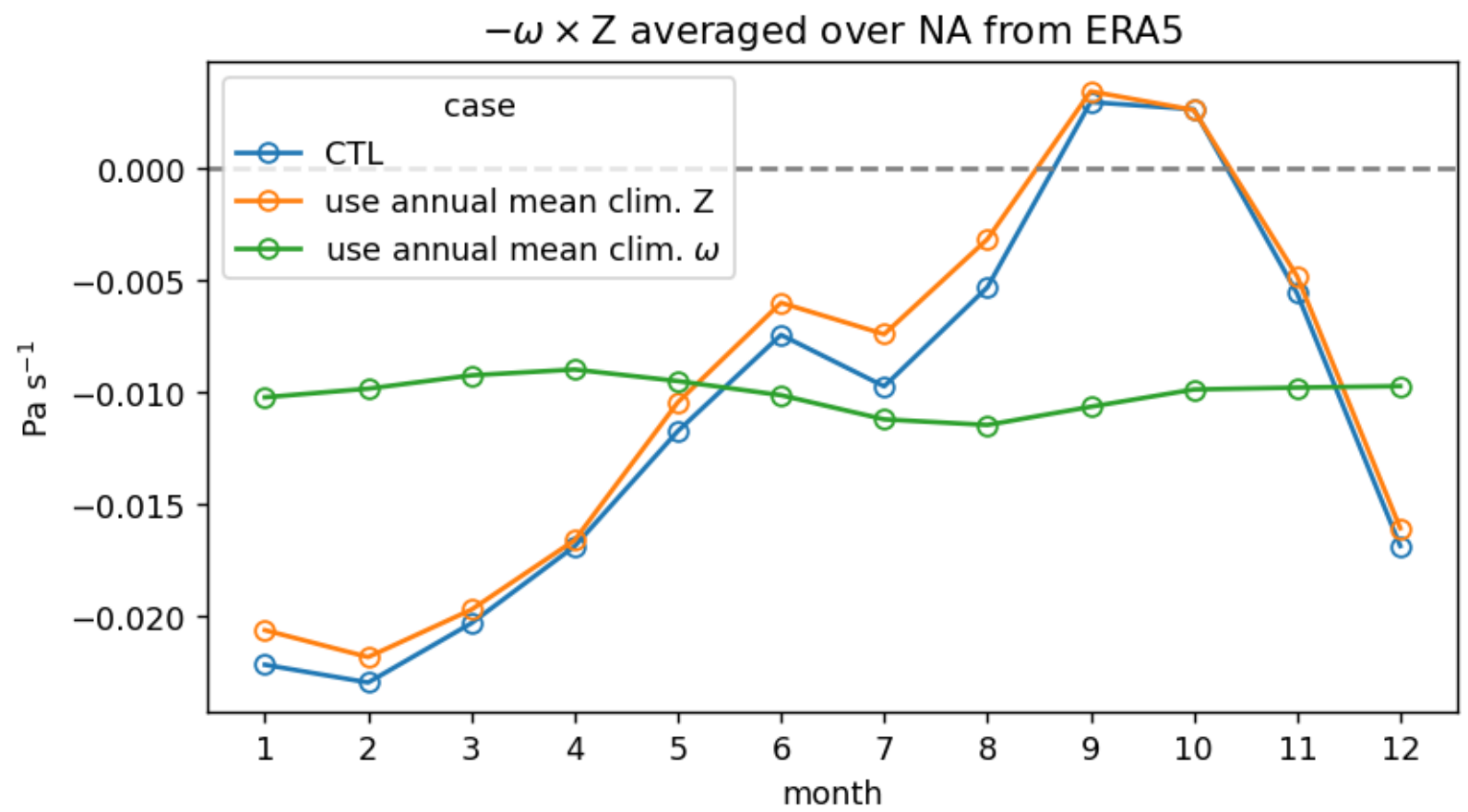

Fig. S12: Seed genesis index of $-\omega \times \mathrm{Z}$ (11) averaged over the NA basin estimated from ERA5 reanalysis, where $\omega$ is the vertical motion velocity in the pressure coordinate (negative upward) and $\mathrm{Z}$ is the ratio of two characteristic length scales (11). Full monthly $\omega$ and $\mathrm{Z}$ are used in the CTL case (blue line), while annual mean climatology of $\omega$ or $Z$ is used in the other two cases (orange and green lines).
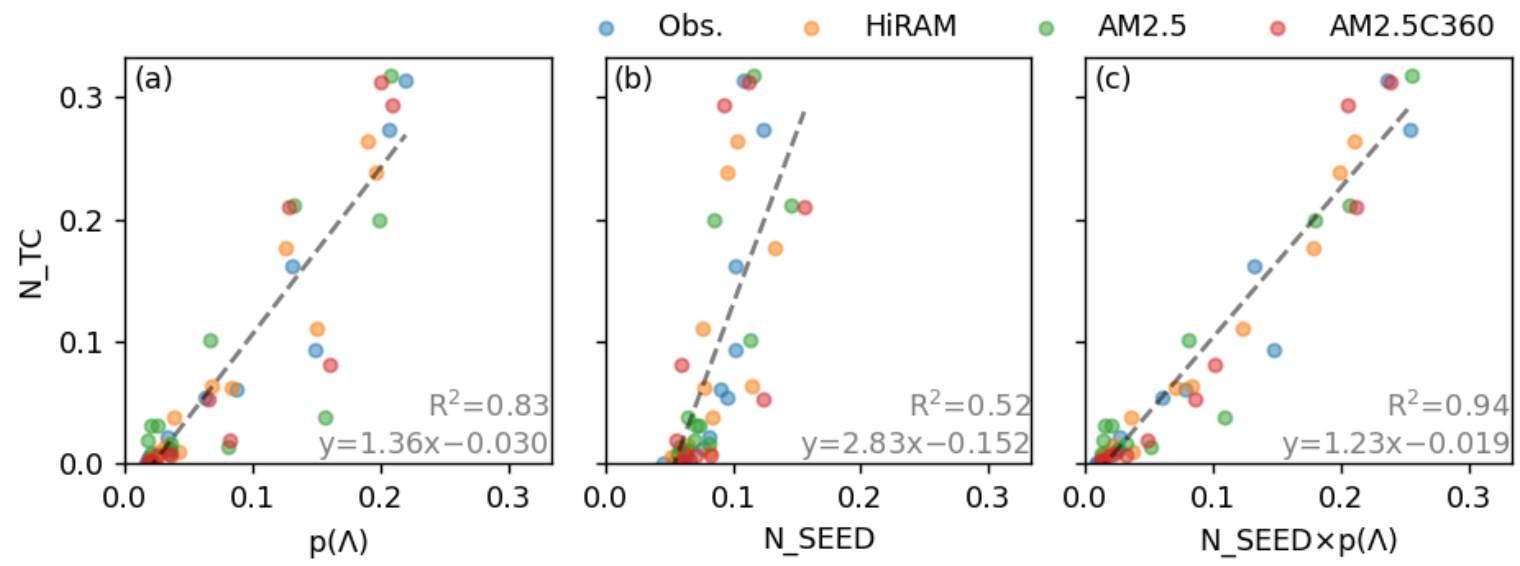

Fig. S13: Same as Fig. 3 except the vorticity threshold in seed tracking decreased by $50 \%$. 

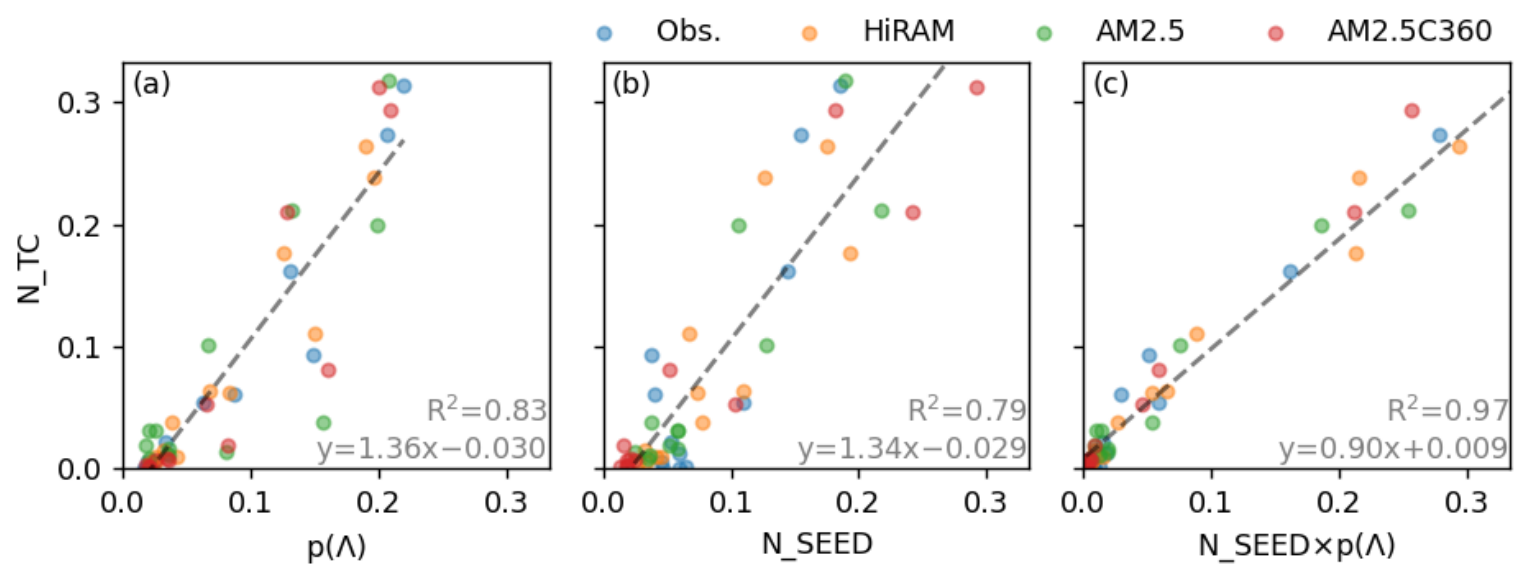

Fig. S14: Same as Fig. 3 except the vorticity threshold in seed tracking increased by $50 \%$.
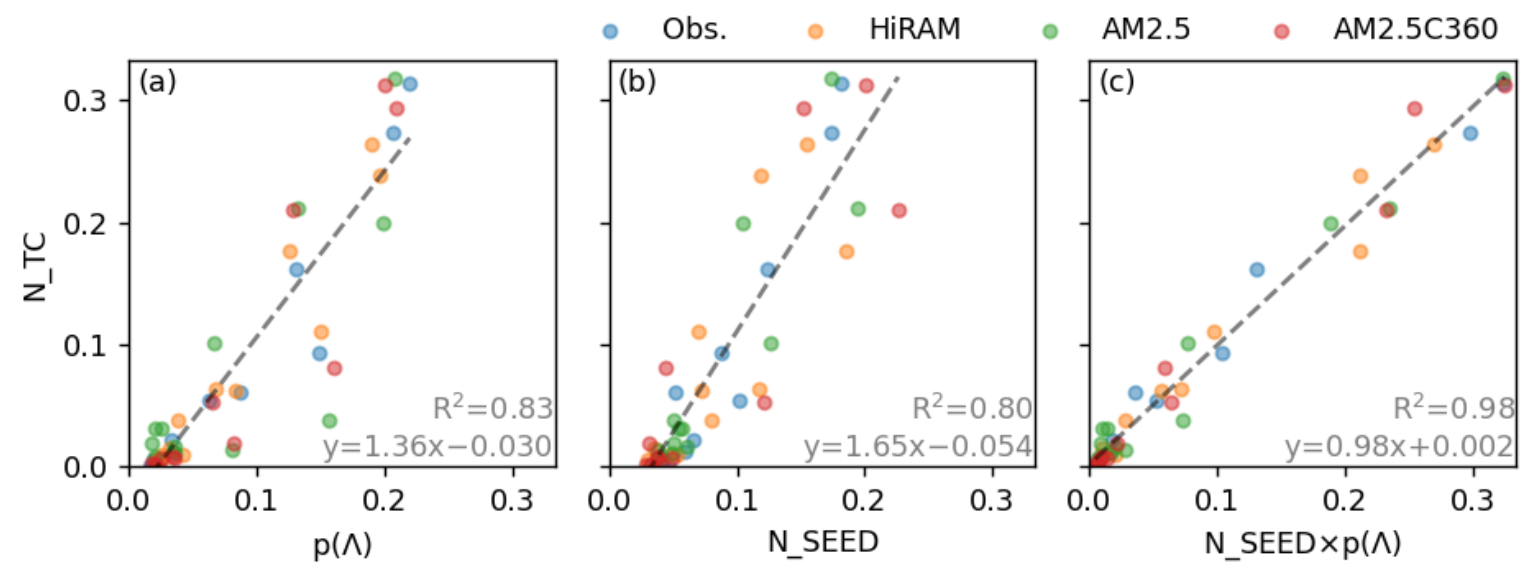

Fig. S15: Same as Fig. 3 except the threshold of ocean hours in seed tracking increased from 12 to 24 hours. 

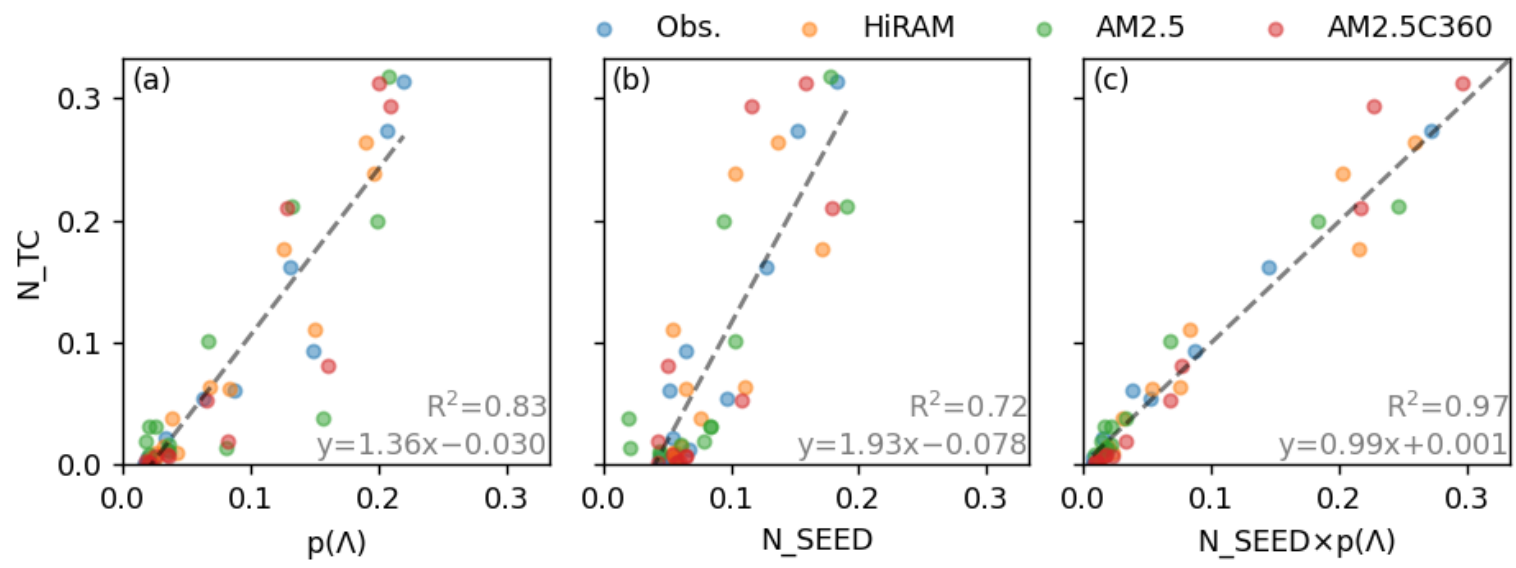

Fig. S16: Same as Fig. 3 except seed tracking using the SLP based algorithm.
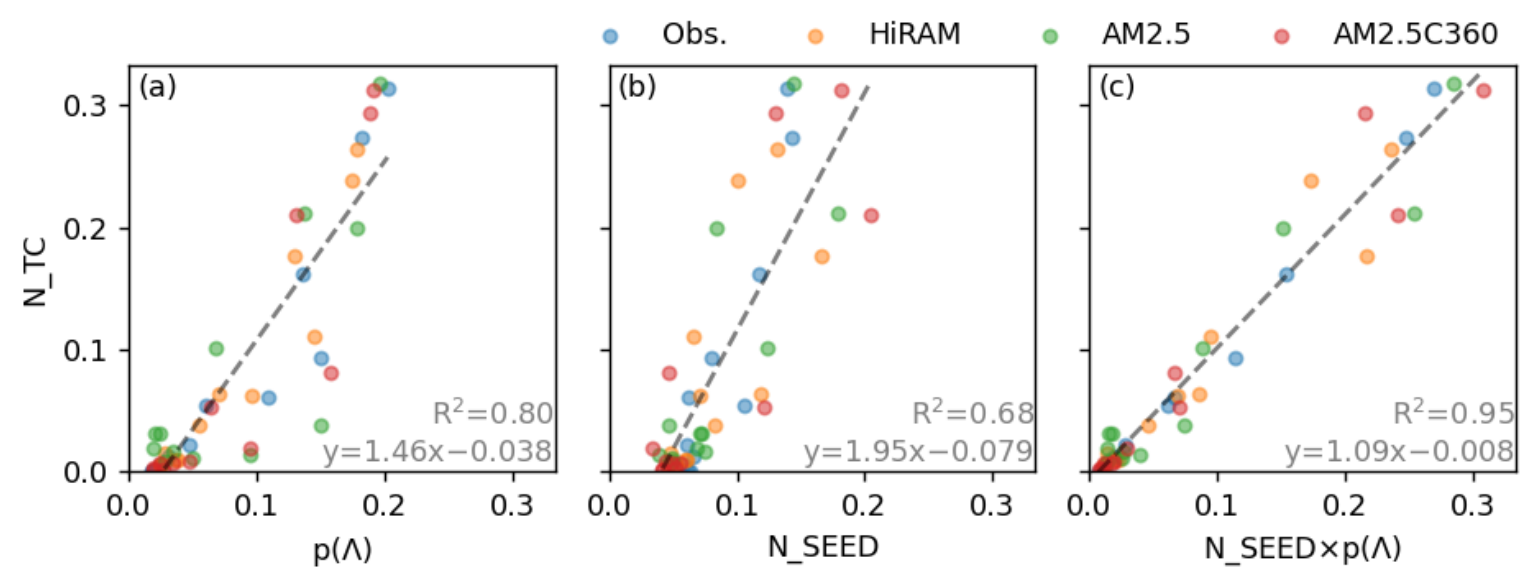

Fig. S17: Same as Fig. 3 except genesis probability averaged poleward of $5^{\circ} \mathrm{N}(\mathrm{S})$ instead of $10^{\circ} \mathrm{N}(\mathrm{S})$. 